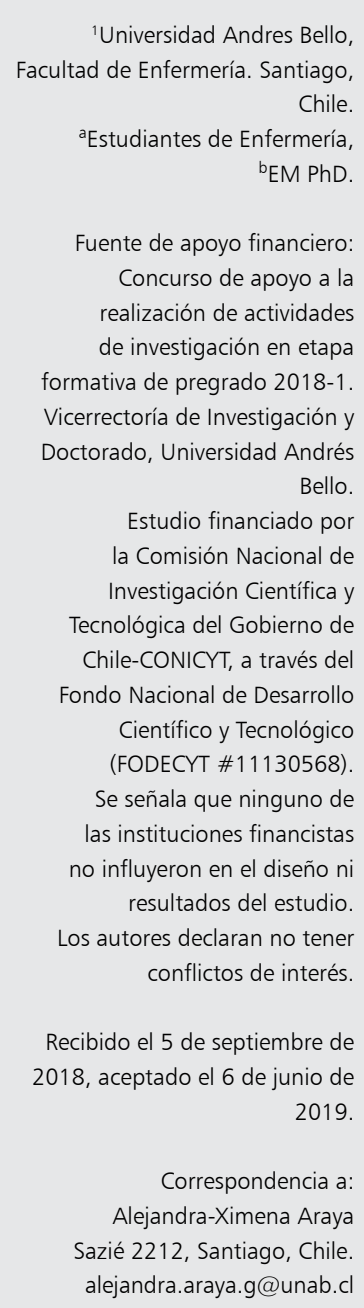

'Universidad Andres Bello, Facultad de Enfermería. Santiago, Chile. aEstudiantes de Enfermería, ${ }^{b} E M P h D$

Fuente de apoyo financiero: Concurso de apoyo a la realización de actividades de investigación en etapa formativa de pregrado 2018-1. Vicerrectoría de Investigación y Doctorado, Universidad Andrés Bello. Estudio financiado por la Comisión Nacional de Investigación Científica y Tecnológica del Gobierno de Chile-CONICYT, a través del Fondo Nacional de Desarrollo Científico y Tecnológico (FODECYT \# 11130568). Se señala que ninguno de las instituciones financistas no influyeron en el diseño ni resultados del estudio. Los autores declaran no tener conflictos de interés.

Recibido el 5 de septiembre de 2018, aceptado el 6 de junio de 2019.

Correspondencia a: Alejandra-Ximena Araya Sazié 2212, Santiago, Chile. alejandra.araya.g@unab.cl

\section{Fragilidad y su correlación con calidad de vida y utilización de los servicios de salud en personas mayores que viven en la comunidad}

\author{
MARÍA PAZ ZÚÑIGA ${ }^{1, a}$, RODRIGO GARCÍA ${ }^{1, a}$, \\ ALEJANDRA XIMENA ARAYA ${ }^{1, b}$
}

\section{Quality of life among frail older people}

Background: Frailty has a great impact in the wellbeing of older people. Aim: To evaluate the quality of life of older people with and without frailty. Material and Methods: We assessed sociodemographic variables, health, integral geriatric assessment, quality of life using the WHOQoL-BREF questionnaire and the level of fragility using the Tilburg Frailty Indicator (TFI) in 538 participants. Results: Three hundred and five participants aged $73 \pm 7$ years (229 women) were classified as fragile and 233 aged $72 \pm 6$ years (125 women) as not having frailty. Compared with their non-fragile counterparts, frail participants had a lower number of years attending school (5.9 and 7.4 respectively), a lower Barther index (93.6 and 98.3 respectively), a lower mini mental score (21.9 and 22.8 respectively) and a higher Yessavage depression score (2.0 and 0.8 respectively). Also, frail participants had a significantly lower total quality of life score and significantly lower scores in the physical and psychological domains. No differences were observed for the social and environmental domains. Older frail participants used health services more frequently than their non-fragile counterparts. Conclusions: In this sample, frailty was associated with a lower quality of life and worse scores in several geriatric assessment tools.

(Rev Med Chile 2019; 147: 870-876)

Key words: Aged; Frailty; Quality of Life.

\begin{abstract}
$\mathrm{C}$ hile, según información del Instituto Nacional de Estadísticas (INE), se ha transformado en un país que continuará envejeciendo a un ritmo acelerado; para el año 2025 habrá 103 personas mayores (PM) por cada cien menores de 15 años ${ }^{1}$. Se estima que para el 2020 , la población mayor será $20 \%$ de la población total del país ${ }^{2}$. Dado este envejecimiento acelerado, es necesario conocer aspectos de la calidad de vida de las PM. La calidad de vida, según la Organización Mundial de la Salud (OMS), ha sido definida como "la percepción del individuo sobre su posición en la vida, en el contexto de la cultura y el sistema de valores en que vive, en relación con sus objetivos,
\end{abstract}

expectativa, estándares y preocupaciones”. Esta definición centra la atención en variables como físicas, psicológicas, relaciones sociales y el ambiente como características esenciales que definen la calidad de vida. Hay varios factores que influyen la calidad de vida de las PM, siendo la fragilidad uno de ellos.

Fragilidad puede ser definido como un fenómeno multidimensional, involucrando aspectos físicos, cognitivos y sociales, y a su vez dinámico ${ }^{4,5}$, el cual puede afectar la calidad de vida de las PM. Particularmente, la fragilidad social se ha relacionado a una reducción en la calidad de la atención prestada a los ancianos frágiles. Junto con lo ante- 
rior, aquellas PM que presentan una peor calidad de vida y estado de fragilidad consultan más a los servicios de atención primaria ${ }^{6-8}$.

Existe poca evidencia científica Latinoamericana que describa la correlación entre fragilidad multidimensional y calidad de vida de los PM que viven en la comunidad. El objetivo de este estudio es analizar la relación entre fragilidad, calidad de vida y utilización de los servicios de atención primaria de las PM que viven en la comunidad en dos comunas de Santiago.

\section{Materiales y Métodos}

Estudio analítico observacional realizado en 538 PM de 60 años que viven en la comunidad, usuarios de seis centros de atención primaria de salud de dos comunas de Santiago, Chile. Las PM elegibles fueron seleccionadas aleatoriamente de bases de datos Servicio de Orientación Médico Estadístico (SOME) del para obtener una muestra representativa de la población de referencia. Los criterios de exclusión fueron: 1) diagnóstico médico de demencia y/o enfermedad terminal e 2) incapacidad de contestar el cuestionario. El tamaño de muestra se determinó para encontrar diferencias entre los frágiles y no frágiles en cuanto a: Calidad de vida y utilización de los servicios de salud, considerando una potencia de $80 \%$, un nivel de la significancia de $95 \%$.

Calidad de vida fue medido con el instrumento WHOQoL-BRIEF, el cual ha sido validado en población mayor chilena ${ }^{9}$. Mide seis dimensiones (habilidades sensoriales, autonomía, actividades del pasado, presente y futuras, participación social, muerte y el morir e Intimidad) con 24 ítems, escala de Likert de 5 puntos. Alpha 0,83 en población chilena ${ }^{9,10}$. El instrumento Frailty Tilburg Indicator (TFI) fue utilizado para medir fragilidad (13 ítems). Las preguntas son dicotómicas y el score de fragilidad se obtiene de la suma de sus preguntas. El rango va de 0-15; es considerado frágil la PM que tenga 5 puntos o más. TFI ha mostrado buenas propiedades psicométricas ${ }^{8,11,14}$, mide los tres tipos de fragilidad y es fácil de auto-aplicar ${ }^{15}$. En esta muestra el alpha fue de 0,86 para el puntaje total y un análisis factorial de $\mathrm{KMO}=0,843$, se obtuvo en chi-cuadrado de 1381.113 con 105 grados de libertad y un valor $\mathrm{p}<0,005$. Utilización de ser- vicios de salud. Se midió a través del número de hospitalizaciones y número de cuidados formales o informales recibidos en el último año. Dentro de la medición de la Valoración geriátrica integral, la capacidad funcional fue medida por la Escala de Barthe ${ }^{16}$ para las actividades de la vida diaria y Escala de Lawton ${ }^{17}$, para las actividades instrumentales de la vida diaria. El MMSE de Folstein fue utilizado para medir el estado cognitivo de la PM. El MMSE tiene un rango total de puntuación del 0-30, donde puntajes más altos indican mejor función cognitiva. Las puntuaciones $<24$ puntos se clasificaron como deterioro cognitivo ${ }^{18}$. Los sintomas depresivos fueron medidos a través de la escala de Yesavage (GDS), versión acortada, de 5 ítems (Sí/No), la cual ha sido diseñada específicamente para la detección de depresión en población adulta mayor. Puntuación $\geq 2$ indica una posible depresión. Esta versión ha sido validada por Hoyl y colaboradores en población chilena ${ }^{19}$ mostrando buenas propiedades psicométricas (Alpha 0,80). El grado de soledad se midió mediante la Escala de Soledad de Huges, que mide aislamiento social a través de 3 ítems, Likert de tres puntos (casi nunca/algunas veces/ muchas veces), que ha demostrado buenas propiedades psicométricas con un alpha de $0,72^{20}$. El grado de apoyo social fue medido a través de la escala multidimensional de percepción de apoyo social (MSPSS) de Zimet y colaboradores ${ }^{21}$. Esta es una escala de 12 ítems que mide apoyo social percibido por los individuos en tres áreas: familia, amigos y otros significativos, la cual ha sido validada en población de PM chilena, reportando un alpha de $0,88^{22}$. Además, se consultó respecto a percepción del estado de salud, número de medicamentos y hospitalización en el último año. Comorbilidad fue medida por el número de enfermedades crónicas auto-reportadas siendo la presencia de dos o más enfermedades considerada como comorbilidad ${ }^{23}$.

\section{Análisis de datos}

Se llevó a cabo un análisis descriptivo de medias, medianas, percentiles, desviaciones estándar $\mathrm{y}$ frecuencias absolutas-relativas para las variables cuantitativas y porcentajes para las variables nominales. Dentro del análisis analítico de variables continuas se usó t de Student para muestras independientes y chi cuadrado para variables dicotómicas según corresponda. 


\section{Aspectos éticos}

Este proyecto de investigación fue aprobado por el Comité de Ética del Servicio de Salud Metropolitano Sur Oriente de Santiago de Chile. Cada una de las personas encuestadas firmó un consentimiento informado.

\section{Resultados}

\section{Descripción socio-demográficas y de salud de la} muestra en estudio

Del total de la muestra $56,69 \%(n=305)$ fue clasificado como frágil según los criterios de fragilidad multidimensional. La Tabla 1 muestra las diferencias en las características socio-demográficas y de salud según fragilidad. La mayoría de los PM frágiles tiene baja escolaridad (5,9 $\pm 3,7$ años) en comparación a los no frágiles ( $7,4 \pm 3,9$ años); son mayoritariamente viudos/as $(31,1 \%)$, mientras que los no frágiles no $(21,5 \%)$. En relación a su estado de salud, la mayoría de las PM frágiles la califican de mala o muy mala $(53,4 \%)$ mientras que los no frágiles califican su salud como muy buena $(75,5 \%)$.

El 63\% de la muestra fueron independientes en sus AVD según escala de Barthel, con un promedio de 7,1 $\pm 1,4$ en las AIVD según el Índice de Lawton. En el área cognitiva, $86 \%$ tienen un MMSE normal y $64 \%$ tiene un test de Yesavage dentro de rangos normales. En relación a aspectos sociales, el grado de soledad fue de 4,6 $\pm 1,9$ puntos en la Escala de Huges (mínimo de 3 y máximo de 9) y el grado de apoyo social fue de $34,6+8,8$ puntos (mínimo de 13 y máximo de 48).

\section{Calidad de vida}

Las personas mayores frágiles reportan diferencias en el puntaje total de calidad de vida, $t$ $(-9,3), \mathrm{df}=536, \mathrm{p}=0,020$ es decir, que el grupo de personas mayores no frágiles reportan una mayor calidad de vida que aquellas frágiles. En la Tabla 2, se muestra los 4 dominios más el puntaje total del cuestionario obtenidos por ambos grupos (frágiles y no frágiles). El puntaje total de calidad de vida obtenido en ambos grupos de personas mayores demostró una significancia estadística $t$ $(-9,3), \mathrm{df}=536, \mathrm{p}=0,020$, reportando una peor evaluación de calidad de vida el grupo de PM frágiles versus aquellas que no lo son.

En relación con los 4 dominios que componen el instrumento de calidad de vida se observó una diferencia significativa en los dominios físico $t$ $(-13,4), \mathrm{df}=536, \mathrm{p}=0,001)$ y psicológico $(t(-9,9)$, $\mathrm{df}=536, \mathrm{p}=0,001)$. Los dominios, relaciones sociales $(0,345)$ y ambiente $(0,081)$, no se evidencia una diferencia estadística significativa.

\section{Utilización de los servicios de salud}

La utilización de los servicios de salud para frágiles y no frágiles, se presentan en la Tabla 3. De total de la muestra, $14,31 \%(\mathrm{n}=77)$ ha estado hospitalizado; $38,10 \%(\mathrm{n}=205)$ han recibido asistencia profesional; $y 24,16 \%(n=130)$ ha requerido ayuda de otras personas para cuidarse en los últimos 12 meses. Las PM frágiles evidencian

Tabla 1. Caracterización de las variables sociodemográficas y del estado de salud de las PM que viven en la comunidad $(n=538)$

\begin{tabular}{|c|c|c|c|c|}
\hline \multirow[t]{2}{*}{ Variables } & \multicolumn{2}{|c|}{ Frágiles $(n=305)$} & \multicolumn{2}{|c|}{ No frágiles $(n=233$ ) } \\
\hline & Promedio (DS) & Porcentaje (n) & Promedio (DS) & Porcentaje (n) \\
\hline \multicolumn{5}{|l|}{ Variables socio-demográficas } \\
\hline Edad en años & $72,7(6,6)$ & & $71,5(5,6)$ & \\
\hline Mujer & & $75,1(229)$ & & $53,9(125)$ \\
\hline Viudo* & & $31,1 \quad(95)$ & & $21,5 \quad(50)$ \\
\hline Años de escolaridad* & $5,9(3,7)$ & & $7,4(3,9)$ & \\
\hline \multicolumn{5}{|l|}{ Variables del estado de salud } \\
\hline Mala/muy mala percepción estado salud* & & $53,4(163)$ & & $14,2 \quad(33)$ \\
\hline Hospitalización en el último año & & $76,3 \quad(58)$ & & $23,7 \quad(18)$ \\
\hline Presencia de enfermedades crónicas & & $93,8(286)$ & & 84,5 (197) \\
\hline
\end{tabular}

*Variables estadísticamente significativas según t de Student para variables continuas o tablas de contingencia para variables categóricas. 
Tabla 2. Descripción de la calidad de vida según fragilidad de la persona mayor

\begin{tabular}{|lccccc|}
\hline $\begin{array}{l}\text { Instrumento de calidad } \\
\text { de vida }\end{array}$ & $\begin{array}{c}\text { Frágil } \\
\text { media } \pm \text { ds }\end{array}$ & $\begin{array}{c}\text { No Frágil } \\
\text { media } \pm \text { ds }\end{array}$ & df & t & p \\
\hline Dominio físico & $20,794,53$ & $25,653,68$ & 536 & $-13,4$ & $0,001^{*}$ \\
\hline Dominio psicológico & $19,584,39$ & $23,013,38$ & 536 & $-9,9$ & $0,001^{*}$ \\
\hline Dominio relaciones sociales & $9,842,24$ & $10,592,03$ & 536 & $-4,0$ & 0,35 \\
\hline Dominio ambiental & $26,025,30$ & $28,514,71$ & 536 & $-5,7$ & 0,08 \\
\hline Puntaje total calidad de vida & $76,7215,75$ & $87,7810,39$ & 536 & $-9,3$ & $0,02^{*}$ \\
\hline
\end{tabular}

Tabla 3. Diferencias de uso de los servicios de salud y fragilidad entre personas mayores frágiles y no-frágiles

\begin{tabular}{|c|c|c|c|c|c|c|}
\hline & \multicolumn{2}{|c|}{ Frágil } & \multicolumn{2}{|c|}{ No frágil } & \multirow[b]{2}{*}{$\chi^{2}$} & \multirow[b]{2}{*}{$\mathbf{p}$} \\
\hline & $\mathbf{n}$ & $\%$ & $\mathbf{n}$ & $\%$ & & \\
\hline ¿Ha estado hospitalizado en el último año? & 58 & 43,7 & 19 & 24,7 & 12,7 & 0,001 \\
\hline ¿Ha recibido cuidado profesional en el último año? & 128 & 62,4 & 77 & 37,6 & 4,4 & 0,039 \\
\hline ¿Ha recibido cuidados informales este año? & 104 & 80,0 & 26 & 20,0 & 37,9 & 0,001 \\
\hline
\end{tabular}

más hospitalizaciones con 19\% más versus los no frágiles.

En relación con la necesidad de cuidados profesionales, las PM frágiles reportan 58\% más de atenciones que sus contrapartes no frágiles. En cuanto al profesional de quién han recibido esos cuidados, las PM frágiles refieren $78,1 \%(\mathrm{n}=100)$ que ha sido médico; $14,1 \%(\mathrm{n}=18)$ un profesional de enfermería; 13,3 por psicólogo; 7,8\% $(\mathrm{n}=10)$ por nutricionista; 7,8\% $(\mathrm{n}=17)$; kinesiólogo; 3,3 $(\mathrm{n}=10)$ y el resto por otros profesionales. En contraste, las PM no frágiles reportan que han sido atendidas por un médico $87,1 \%(\mathrm{n}=67)$; $16,9 \%(\mathrm{n}=13)$ un profesional de enfermería; 9,1\% $(\mathrm{n}=7)$ por nutricionista; $3,89(\mathrm{n}=3)$ por kinesiólogo; 10,4\% $(\mathrm{n}=8)$; por psicólogo/a y el resto por otros profesionales. Cuando se pregunta con qué frecuencia ha visitado al médico en el último año, destacan los frágiles con $33,4 \%$ versus los no frágiles que indican $15,5 \%$, en la alternativa "más de 7 veces".

Respecto a los cuidados informales y su necesidad, frágiles indican que sí los han recibido en $34,1 \%(\mathrm{n}=104)$, mientras que no frágiles $11,2 \%$ $(\mathrm{n}=26)$. Al identificar la persona que ayuda en el cuidado, se a un familiar con 93,3\% ( $=97)$; un vecino con $8,7 \%(n=9) \%$; y el resto otros para
PM frágiles. Mientras que las personas no frágiles solo identifican a un familiar como aquella persona que ayuda en su cuidado en caso de necesitarlo

Del total de la muestra, en caso de emergencia, ambos grupos refieren tener a quién recurrir en caso de presentar problemas de salud, frágiles $96,7 \%(\mathrm{n}=295)$ y no frágiles $99,1 \%(\mathrm{n}=231)$. La ayuda provendría de sus familiares como principal red de apoyo frente a una emergencia, frágiles con $88,9 \%(\mathrm{n}=271)$ y no frágiles $96,1 \%(\mathrm{n}=224)$; seguidos de vecinos $23,3 \%(n=71)$ frágiles vs $30 \%$ $(\mathrm{n}=30)$ para no frágiles; grupos organizados $5,2 \%$ $(\mathrm{n}=16)$ frágiles vs $14,6 \%(\mathrm{n}=34)$ no frágiles; $y$ finalmente $3 \%$ de PM frágiles $(n=9)$ señalan que esta ayuda podría ser entregada por el CESFAM y no frágiles marcan $2,6 \%(n=6)$ para el mismo proveedor de ayuda.

\section{Discusión}

Las PM frágiles presentan puntajes más bajos en las ABVD e AIVD, deterioro cognitivo, y presentan mayor puntaje en el tamizaje de depresión que sus contrapartes no frágiles. En este estudio se evidencia diferencias significativas en la calidad de vida entre personas mayores frágiles y no frágiles, 
estableciendo una correlación inversa con el estado de fragilidad ${ }^{24}$. Estas diferencias son observables sólo en los dominios físico y psicológicos del instrumento de calidad de vida.

Los resultados de los dominios físico y psicológico son similares a los obtenidos en otros países, siendo las áreas más críticas dentro del primer dominio mencionado, aquellas relacionadas con el dolor físico, la satisfacción con el sueño, desplazamiento $^{24}$, la satisfacción con la capacidad de trabajo ${ }^{25}$ y la necesidad de algún tipo de tratamiento médico ${ }^{24}$. Para el dominio psicológico, el área crítica corresponde a los sentimientos negativos como tristeza y depresión, estableciéndose similitud con otros estudios que señalan que este dominio puede ser un buen predictor de calidad de vida ${ }^{26,27}$.

Los dominios de relaciones sociales y ambiente no se observaron diferencias significativas. En cuanto al dominio social, los resultados de la muestra, difieren de la literatura que indica a éste como uno de los más afectados en relación a la calidad de vida ${ }^{25}$. Una posible explicación a esto puede estar dado por un mayor desarrollo de las redes de apoyo de estos adultos mayores, según el análisis de los resultados a las preguntas que evalúan la utilización de los servicios de salud, pudiendo esta idea ser analizada en mayor profundidad en un futuro estudio.

En relación a la utilización de los servicios de salud, se observa en la población frágil, una mayor utilización de los mismos. Estos resultados se condicen según la literatura, y está dado mayoritariamente por las características que componen el perfil de la persona mayor frágil. En este sentido, intervenciones preventivas deben incluir no solo los aspectos físicos considerados en la definición de fragilidad, sino que también aquellos psicológicos y sociales. Según Lenardt' et al, "la fragilidad en los ancianos no es igual a un resultado de mala calidad de vida. La fragilidad es una condición manejable que puede ser un objetivo a través de intervenciones de enfermería geriátrica" ${ }^{24}$.

Llama la atención el alto número de personas clasificadas como frágiles en este estudio. Una de las explicaciones para este hallazgo puede ser que el instrumento utilizado para clasificarlos como frágiles, utiliza la definición multidimensional del síndrome, lo que incluye aspectos psicológicos y sociales sumado a lo físico y, como consecuencia, el porcentaje es elevado en comparación con es- tudios que solo miden la fragilidad física. En este sentido, la evidencia publicada sobre fragilidad en Chile es de Alvarado y colaboradores ${ }^{29}$. Este grupo de investigadores reportaron $43 \%$ de fragilidad física en Santiago en el año 1999-2000 medida con los criterios de fragilidad física de Lindra Fried. Basado en lo anterior, el porcentaje de fragilidad física de personas mayores era alto $y$, en consecuencia, si se utiliza un instrumento, como el TFI que mide fragilidad física, psicológica y social, es esperable que ese porcentaje aumente y, podría explicar, el porcentaje de fragilidad reportado en este estudio.

La prevalencia de fragilidad es variada a nivel mundial ya que depende de varios factores como, por ejemplo, definición de fragilidad, tipo de muestra e instrumento de medición que se emplee. $\mathrm{Si}$ se hace una búsqueda de estudios similares a este, es decir, resultados de investigación que han medido la fragilidad multidimensional con el instrumento TFI en PM que viven en la comunidad, los porcentajes varían entre $51 \%$ a $58 \%{ }^{30-34}$; en consecuencia, el porcentaje de fragilidad encontrado en este estudio concuerda con lo encontrado en la literatura internacional. Dentro de las limitaciones de esta investigación se encuentra que no se pueden establecer causalidad entre las variables en estudio por el tipo de diseño del mismo. Además, la muestra fue seleccionada de los listados de PM adscritas (pasivas y activas) de seis CESFAM de dos comunas vulnerables de Santiago. Dentro de sus fortalezas que en la selección de la muestra fue aleatoria de forma de minimizar el sesgo de selección de la misma.

En conclusión, las PM frágiles de este estudio presentan peor calidad de vida y utilizan con mayor frecuencia los servicios de salud que sus contrapartes no frágiles. Es urgente la necesidad de que las intervenciones en el área salud que sean preventivas, siguiendo el modelo chileno de atención integral de salud familiar y comunitaria ${ }^{28} \mathrm{de}$ forma de prevenir la dependencia fomentando un envejecimiento saludable en las personas mayores que viven en la comunidad.

\section{Referencias}

1. Pontificia Universidad Católica de Chile, Instituto de Sociología UC. Chile y sus mayores. Resultados de la segunda encuesta de calidad de vida 2010. Santiago: Pon- 
tificia Universidad Católica de Chile; 2011. http://www. senama.gob.cl/storage/docs/Resultados-Segunda-Encuesta-Nacional-Calidad-de-Vida-en-la-Vejez-2011.pdf.

2. Instituto Nacional de Estadísticas. Enfoque Estadístico - Adulto Mayor en Chile, Boletín informativo del Instituto Nacional de Estadística 2007 Sep.; 1-4 https:// www.ine.cl/docs/default-source/FAQ/enfoque-estad\%C3\%ADstico-adulto-mayor-en-chile.pdf?sfvrsn=2.

3. Espinoza I, Osorio P, Torrejón M J, Lucas-Carrasco R, Bunout D. Validación del cuestionario de calidad de vida (WHOQOL-BREF) en adultos mayores chilenos. Rev Med Chile 2011; 139 (5): 579-86.

4. Gobbens RJ, Luijkx KG, Van Assen MA. Explaining quality of life of older people in the Netherlands using a multidimensional assessment of frailty. Qual Life Res 2012; 22: 2051-61.

5. Luis Ramos GE, Libre Rodríguez J de J. Fragilidad en el adulto mayor: Un primer acercamiento. Rev Cubana Med Gen Integr 2004; 20(4).

6. Gobbens RJ, van Assen MA. Frailty and its prediction of disability and health care utilization: the added value of interviews and physical measures following a self-report questionnaire. Arch Gerontol Geriatr 2012; 55 (2): 36979.

7. Gobbens RJ, van Assen MA, Luijkx KG, Schols JM. The predictive validity of the Tilburg Frailty Indicator: disability, health care utilization, and quality of life in a population at risk. Gerontologist 2012; 52 (5): 619-31.

8. Gobbens RJ, van Assen MA, Luijkx KG, Wijnen-Sponselee MT, Schols JM. The Tilburg Frailty Indicator: psychometric properties. J Am Med Dir Assoc. [Validation Studies] 2010; 11 (5): 344-55.

9. Espinoza I, Osorio P, Torrejón M, Lucas-Carrasco R, Bunout D. Validación del cuestionario de calidad de vida (WHOQOL-BREF) en adultos mayores chilenos. Rev Med Chile 2011; 138: 579-86.

10. Urzúa A, Navarrete M. Calidad de vida en adultos mayores: análisis factoriales de las versiones abreviadas del WHOQoL-Old en población chilena. Rev Med Chile 2013; 141: 28-33.

11. Daniels R, van Rossum E, Beurskens A, van den Heuvel $\mathrm{W}$, de Witte L. The predictive validity of three self-report screening instruments for identifying frail older people in the community. BMC Public Health 2012; 12: 69.

12. Bilotta C, Nicolini P, Case A, Pina G, Rossi S, Vergani C. Frailty syndrome diagnosed according to the Study of Osteoporotic Fractures (SOF) criteria and adverse health outcomes among community dwelling older outpatients in Italy. A one-year prospective cohort study. Arch Gerontol Geriatr 2012; 54 (2): e23-8.
13. Metzelthin SF, Daniels R, van Rossum E, de Witte L, van den Heuvel WJ, Kempen GI. The psychometric properties of three self-report screening instruments for identifying frail older people in the community. BMC public health 2010; 10: 176.

14. Gobbens RJ, van Assen MA, Luijkx KG, Wijnen-Sponselee MT, Schols JM. Determinants of frailty. J Am Med Dir Assoc 2010; 11 (5): 356-64.

15. Comité Internacional de Directores de Revistas Médicas. Requisitos Uniformes para preparar los manuscritos que se presentan a las revistas biomédicas: redacción y edición de las publicaciones biomédicas. Rev Panam Salud Pública 2004; 15 (1): 41-57.

16. Mahoney FI, Barthel DW. Functional Evaluation: The Barthel Index. Md State Med J 1965; 14: 61-5.

17. Lawton MP, Brody EM. Assessment of older people: self-maintaining and instrumental activities of daily living. Gerontologist 1969; 9 (3): 179-86.

18. Folstein MF, Folstein SE, McHugh PR. "Mini-mental state". A practical method for grading the cognitive state of patients for the clinician. J Psychiatr Res 1975; 12 (3): 189-98.

19. Hoyl MT, Alessi CA, Harker JO, Josephson KR, Pietruszka FM, Koelfgen M, et al. Development and testing of a five-item version of the Geriatric Depression Scale. J Am Geriatr Soc 1999; 47 (7): 873-8.

20. Hughes ME, Waite LJ, Hawkley LC, Cacioppo JT. A Short Scale for Measuring Loneliness in Large Surveys: Results From Two Population-Based Studies. Res Aging 2004; 26 (6): 655-72.

21. Zimet GD, Dahlem NW, Zimet SG, Farley GK. The Multidimensional Scale of Perceived Social Support. J Pers Assess 1988; 52 (1): 30-41.

22. Arechabala Mantuliz MC, Miranda Castillo C. Validación de una escala de apoyo social percibido en un grupo de adultos mayores adscritos a un programa de hipertensión de la Región Metropolitana. Cienc Enferm 2002; 8 (1): 49-55.

23. Sousa AC, Dias RC, Maciel AC, Guerra RO. Frailty syndrome and associated factors in community-dwelling elderly in Northeast Brazil. Arch Gerontol Geriatr 2012; 54 (2): e95-e101.

24. Lenardt M, Carneiro NHK, Binotto MA, Willig MH, Lourenço TM, Albino, J. Frailty and quality of life in elderly primary health care users. Rev Bras Enferm 2016; 69 (3): 448-53.

25. Varela FR, Ciconelli RM, Campolina AG, Soarez PC. Quality of life evaluation of fraily elderly in Campinas, Sao Paulo. Rev Assoc Med Bras 2015; 61 (5): 423-30.

26. Kim HJ, Park S, Park SH, Heo YW, Chang BS, Lee CK, et al. The significance of frailty in the relationship be- 
Fragilidad, calidad de vida y servicios de salud en personas mayores - M. P. Zúñiga et al

tween socioeconomic status and health-related quality of life in the Korean community-dwelling elderly population: mediation analysis with bootstrapping. Quality of Life Reserch 2017; 26 (12): 3323-30.

27. Bilotta $\mathrm{C}$, Bowling $\mathrm{A}$, Casè $\mathrm{A}$, Nicolini $\mathrm{P}$, Mauri $\mathrm{S}$, Castelli $\mathrm{M}$, et al. Dimensions and correlates of quality of life according to frailty status: a cross-sectional study on community-dwelling older adults referred to an outpatient geriatric service in Italy. Health and Quality of Life Outcomes 2010; 8 (56): 1-10.

28. Ministerio de Salud Gobierno de Chile, Subsecretaría de Redes Asistenciales. División de Atención Primaria; Organización Panamericana de la Salud. Orientaciones para la implementación del modelo de atención integral de salud familiar y comunitaria: dirigido a equipos de salud.; pp.8-143. https://www.minsal.cl/portal/url/item/ e7b24eef3e5cb5d1e0400101650128e9.pdf.

29. Alvarado BE, Zunzunegui MV, Beland F, Bamvita JM. Life course social and health conditions linked to frailty in Latin American older men and women. J Gerontol A
Biol Sci Med Sci 2008; 63 (12): 1399-406.

30. Mulasso A, Brustio P, Rainoldi A, Zia G, Feletti L, N'dja A, et al. A comparison between an ICT tool and a traditional physical measure for frailty evaluation in older adults. BMC Geriatr 2019; 19 (1): 88.

31. Verver D, Merten H, de Blok C, Wagner C. A cross sectional study on the different domains of frailty for independent living older adults. BMC Geriatr 2019; 19 (1): 61 .

32. Verver D, Merten H, Robben P et al. Care and support for older adults in The Netherlands living independently. Health Soc Care Community 2018; 26 (3): 404-14.

33. Mulasso A, Argiolu L, Roppolo M, Azucar D, Rabaglietti E. Emotion experience and frailty in a sample of Italian community-dwelling older adults. Clin Interv Aging 2017; 12 (28): 2017-24.

34. Coelho T, Santos R, Paúl C, Gobbens RJ, Fernandes L. Portuguese version of the Tilburg Frailty Indicator: Transcultural adaptation and psychometric validation. Geriatr Gerontol Int 2015; 15 (8): 951-60. 